\title{
Influência de 12 semanas de treinamento excêntrico nas razões de torque concêntrico e excêntrico de flexores/extensores do joelho
}

\author{
Julio Cézar Lima da Silva*, Lucas Coelho Job, Fábio Juner Lanferdini, \\ Clarice Sperotto dos Santos Rocha, Marco Aurélio Vaz
}

\begin{abstract}
Resumo O treinamento excêntrico altera a capacidade de produção de força de maneira diferente entre grupos musculares antagonistas. Razões de torque possibilitam identificar essas adaptações do treinamento excêntrico nos músculos antagonistas. Este estudo investigou a influência do treinamento excêntrico nas razões de torque dos flexores/extensores do joelho. Vinte e dois voluntários foram divididos em um grupo controle $(n=10)$ e outro experimental $(n=10)$. O grupo experimental foi submetido a 12 semanas de treinamento excêntrico na velocidade angular de $-60 \%$ s. As razões de torque foram avaliadas nas velocidades angulares concêntricas de $60 \%$ s e $420 \%$, e excêntricas de $-60 \%$ s e $-300 \%$ s. A razão de torque concêntrico aumentou na velocidade de $420 \%$ do pré para o pós-treinamento devido a uma maior capacidade de geração de torque dos isquiotibiais em relação ao quadríceps no pós-treinamento para essa velocidade. A razão de torque excêntrico em $-60 \%$ s reduziu do pré para o pós-treinamento devido ao maior ganho de torque do quadríceps em relação aos isquiotibiais com o treinamento. O menor comprimento de fibras do quadríceps comparado ao dos isquitibiais parece ter sido responsável pelo aumento de torque excêntrico no pós-treinamento na velocidade angular de treinamento, enquanto o aumento da razão concêntrica na velocidade angular de $420 \%$ parece estar associado ao maior comprimento de fibras dos isquiotibiais, conferindo a esse grupo muscular uma vantagem mecânica em grandes velocidades. Conclui-se que o treinamento excêntrico altera as razões de torque dos músculos antagonistas da articulação do joelho de acordo com as características estruturais de cada grupo muscular.
\end{abstract}

Palavras-chave Treinamento excêntrico, Razões de torque, Articulação do joelho, Quadríceps, Isquiotibiais.

\section{Influence of 12 weeks of eccentric training on knee flexors/extensors concentric and eccentric torque ratios}

\footnotetext{
Abstract Eccentric training changes the force production capacity in different ways between antagonistic muscle groups. Torque ratios allow for the identification of these adaptations of eccentric training between antagonistic muscles. This study investigated the influence of eccentric training on the knee flexors/extensors torque ratios. Twenty-two volunteers were divided into a control $(n=10)$ and an experimental $(n=10)$ group. The experimental group underwent a 12-weeks eccentric training program at an angular velocity of - $60 \%$ s. The torque ratios were evaluated at the concentric angular velocities of $60 \%$ s e $420 \%$ s and eccentric velocities of $-60 \% \mathrm{~s}$ and $-300 \% \mathrm{~s}$. The concentric torque ratio increased at the angular velocity of $420 \% \mathrm{~s}$ from pre to post-training due to an increased torque capacity of the hamstrings compared to that of the quadriceps at this angular velocity post-training. The eccentric torque ratio at $-60 \%$ s reduced from pre to post-training due to the higher increase in the quadriceps torque compared to that of the hamstrings with training. The smaller fiber length of the quadriceps compared to that of the hamstrings seems to have been responsible for the increased eccentric torque at the training velocity post-training, whereas the increase in the concentric torque ratio at the angular velocity of $420 \%$ s seems to be associated to the higher fiber length of the hamstrings, giving this muscle group a mechanical advantage to produce force at high angular velocities. We conclude that eccentric training changes the torque ratios of knee antagonistic muscle groups according to their structural characteristics.
}

Keywords Eccentric training, Torque ratios, Knee joint, Quadriceps, Hamstrings. 


\section{Extended Abstract}

\section{Introduction}

Eccentric training is an important resource in the recovery and prevention of the musculoskeletal system injuries (Pull and Ranson, 2007), in the strengthening of both the contractile and non-contractile structures of skeletal muscles and in promoting neural adaptations (Albert, 2002; Michaut and Pousson, 2004). This type of training is commonly used in physiotherapy treatments to accelerate the rehabilitation or even to increase muscle performance in athletes and non-athletes (Heiderscheit et al., 2010; Jonsson and Alfredson, 2005). Muscle imbalances are a common source of skeletal muscle injury, as one muscle group increases its force production capacity several times above that of the antagonistic muscle group. This may occur after training in sports or after a period of immobilization post-injury, for example. Muscle imbalances might be identified by calculating torque ratios (Hamstrings (H)/Quadriceps (Q)) (Lanferdini et al., 2010; O'sullivan et al., 2008). Normal $H: Q$ ratios at low angular velocities $\left(60^{\circ} / \mathrm{s}\right)$ range between 0.50 and 0.80 (Kannus, 1998, Lephart et al., 2001). The purpose of this study was to investigate the influence of eccentric training on $H: Q$ torque ratios at four (2 concentric and 2 eccentric) different angular velocities.

\section{Material and methods}

Twenty healthy male subjects signed an informed consent form to participate in the study, whose procedures were approved by the University Ethics in Research Committee (Protocol number 2003123). Subjects were assigned either to an experimental group ( $E G ; n=10$; weight $=70.76 \pm 9.99 \mathrm{~kg}$; height $=175.76 \pm 7.31 \mathrm{~cm}$; age $=27.6 \pm 7.09$ years $)$, which underwent a 12-weeks eccentric training program, or to a control group $(C G ; n=10$; weight $=74.72 \pm 8.46 \mathrm{~kg}$; height $=177.62 \pm 5.56 \mathrm{~cm}$; age $=27.1 \pm 4.65$ years .

The 12-weeks of eccentric training program can be seen in Table 1. Load was increased by increasing the number of repetitions and the number of series throughout the program. The right knee of the subjects of both groups (CG and EG) was evaluated with an isokinetic dynamometer (Cybex, NORM model; Lumes \& Co., Ronkonkoma, NY, USA) before and after eccentric training. Knee flexors and extensors torque was measured using three maximal voluntary contractions concentrically $(60 \%$, $420 \% \mathrm{~s})$ and eccentrically $(-60 \% \mathrm{~s},-300 \% \mathrm{~s})$. The torque obtained in the contraction of greater peak value was chosen from each muscle group for data analysis. Prior to the test protocol, subjects performed a warm-up on a cycle ergometer for 5 minutes at a power of 60 watts. They also performed active stretching of the muscle groups involved in training for 20 seconds before and after the torque testing sessions.

The H:Q torque ratio data are presented as mean and standard deviation. Shapiro-Wilk test and Mauchly were used to verify normality and sphericity of the data. A two-way ANOVA (groups, assessments) for repeated measures with post hoc Bonferroni were used to identify differences between pre and post-training effects. The Student t-test for dependent data was used in the comparison between the values of torque (extensors and flexors) before and after eccentric training. Significant differences $\alpha=0.05$.

\section{Results}

In concentric H:Q torque ratios increased from $0.43 \pm 0.14$ pretraining to $0.53 \pm 0.16$ post-training at an angular velocity of $420 \%$ s $(p=0.036)$ (Figure 1), while the eccentric H:Q torque ratios reduced from $0.57 \pm 0.09$ at pre-training to $0.48 \pm 0.07$ at post-training at an angular velocity of $-60 \% \mathrm{~s}(p=0.022)$ (Figure 2). No differences were observed in the concentric and eccentric torque ratios of the control group.

\section{Discussion and conclusion}

The concentric H:Q ratio increase is related to a greater gain of concentric torque of the hamstrings compared to that of the quadriceps at an angular velocity of $420 \%$ s (Table 2). This result might be due to the hamstrings morphology, as they have an intermediate cross-sectional area, long-fibers and a small fascicle pennation angle. These morphological characteristics give this muscle group an advantage to produce force at high contraction velocities (or angular velocities). On the other hand, the reduction observed in the H:Q eccentric ratio at the angular velocity of $-60 \%$ s (Table 2) is associated with a higher eccentric torque gain in the quadriceps muscle compared to that observed for the hamstrings. This increased quadriceps gain in eccentric torque might be due to the large cross-sectional area, large pennation angle and short fibers of this muscle, which make it more effective in producing forces at low contraction and/or angular velocities (Kannus 1998; Lanferdini et al. 2010; Lephart et al. 2001).

Further studies of intrinsic muscle parameters using image techniques such as ultrasound may help to explain the structural changes caused by eccentric training and allow for a better understanding of the mechanisms of skeletal muscle plasticity after eccentric training. 


\section{Introdução}

O treinamento excêntrico é um importante recurso na recuperação e prevenção de lesões do sistema músculoesquelético (Pull e Ranson, 2007). Ele fortalece tanto as estruturas contráteis quanto as não-contráteis, e promove adaptações neurais (Albert, 2002; Michaut e Pousson, 2004). Este tipo de treinamento é comumente utilizado em tratamentos fisioterápicos a fim de acelerar a reabilitação ou mesmo para incremento do desempenho muscular em atletas e não-atletas (Heiderscheit et al., 2010; Jonsson e Alfredson, 2005).

Contudo, sobrecargas mecânicas impostas pelo treinamento excêntrico em grupos musculares com diferentes arquiteturas musculares (diferente arranjo de suas fibras em relação à linha de geração de força do músculo) resultam em alterações nos ganhos de força, provocando desequilíbrio muscular entre músculos antagonistas (Lanferdini et al., 2010; Lieber e Fridén, 2000). Estes possíveis desequilíbrios musculares podem ser identificados por meio do cálculo das razões de torque (Isquiotibiais (I)/Quadriceps (Q)) (Lanferdini et al., 2010; O'sullivan et al., 2008). A investigação destes desequilíbrios musculares antagonistas auxilia no processo de prevenção e tratamento de lesões dos mesmos (Aagaard et al., 1998; Kannus, 1998).

As razões I:Q em velocidades angulares baixas $\left(60^{\circ} / \mathrm{s}\right)$ situam-se entre 0,50 e 0,80 (Kannus, 1998; Lephart et al., 2001). Contudo, em velocidades de contração mais elevadas $(300 \%$ s) o torque produzido pelos flexores do joelho deve ser semelhante ao torque dos extensores do joelho, ou seja, as razões de torque são aproximadas, tanto em sujeitos saudáveis como em sujeitos que sofreram lesões prévias nos isquiotibiais (Appen e Ducan, 1986; O'sullivan et al., 2008). Impellizzeri et al. (2008) encontraram uma razão de torque de 0,53 entre I:Q em contrações excêntricas na velocidade angular de $60 \%$ s, sendo semelhante para as contrações concêntricas em indivíduos saudáveis.

$\mathrm{O}$ fato de diferentes grupos musculares terem diferentes arquiteturas musculares (Lieber e Fridén, 2000) pode resultar em diferentes adaptações e consequentemente alterações na capacidade de produção de força destes músculos após um período de treinamento. Esta adaptação está relacionada à velocidade e a carga com que o treinamento é realizado (Farthing e Chilibeck, 2003). Estudos desenvolvidos com treinamento isocinético (controle da velocidade angular), demostram que diferentes velocidades angulares $\left(30^{\circ} / \mathrm{s}, 60^{\circ} / \mathrm{s}, 90^{\circ} / \mathrm{s}, 180^{\circ} / \mathrm{s}\right)$ provocam diferentes ganhos de força em um mesmo grupo muscular (Farthing e Chilibeck 2003;
Paddon-Jones et al. 2001; Seger e Thorstensson, 2005). Estes fatores tem relação direta com a arquitetura muscular de cada grupo muscular, ou seja, a velocidade de treinamento adotada durante execuções máximas isocinéticas podem definir qual dos grupos musculares antagonistas incrementará mais sua capacidade de produção de força após um período de treinamento.

Considerando que diferentes arquiteturas musculares podem ser responsáveis por determinar diferentes variações de ganho de capacidade de produção de força entre grupos musculares antagonistas, o objetivo deste estudo foi investigar a influência do treinamento excêntrico nas razões de torque de flexores/extensores do joelho (razões entre o torque dos músculos isquiotibiais e do quadríceps; razões I:Q) em diferentes velocidades angulares. Hipotetizamos, assim, que as razões de torque em baixas velocidades sofrerão mais influência dos extensores do joelho devido a um maior ganho de força decorrente de sua grande área de seção transversa fisiológica e fibras curtas, enquanto a vantagem mecânica deverá se inverter nas maiores velocidades angulares onde músculos com fibras longas como os isquiotibiais deverão produzir relativamente mais força do que os extensores do joelho com o treinamento excêntrico.

\section{Materiais e Métodos}

A amostra foi composta por 20 sujeitos do sexo masculino, que assinaram um termo de consentimento para a participação do estudo, cujos procedimentos foram aprovados pelo Comitê de Ética em Pesquisa da Universidade Federal do Rio Grande do Sul (Parecer número 2003123). A amostra foi selecionada de forma intencional e dividida em dois grupos: grupo experimental (GE; $\mathrm{n}=10$; massa corporal $=70,76 \pm 9,99 \mathrm{~kg}$; estatura $=175,76 \pm 7,31 \mathrm{~cm}$; idade $=27,6 \pm 7,09$ anos); e grupo controle $(\mathrm{GC} ; \mathrm{n}=10$; massa corporal $=74,72 \pm 8,46 \mathrm{~kg}$; estatura $=177,62 \pm 5,56 \mathrm{~cm}$; idade $=27,1 \pm 4,65$ anos).

Como critérios de exclusão deste estudo, os sujeitos não deveriam ter histórico de lesões musculares ou articulares nos membros inferiores, disfunções no sistema nervoso central e periférico ou doenças sistêmicas previamente diagnosticadas. Como critérios inclusão no estudo os sujeitos não deveriam estar realizando nenhum tipo de treinamento de força envolvendo a musculatura a ser analisada.

Somente os sujeitos do GE foram submetidos a um programa de treinamento excêntrico durante 12 semanas para os músculos flexores e extensores do joelho, enquanto o GC não realizou qualquer tipo de treinamento nas musculaturas envolvidas nos testes. 


\section{Protocolo de treinamento excêntrico}

O protocolo consistiu de 12 semanas de treinamento excêntrico com uma frequência semanal de três sessões, sendo composta de duas a cinco séries de sete a nove repetições de contrações excêntricas máximas dos grupos musculares flexores e extensores do joelho a uma velocidade angular excêntrica de $-60 \%$ s. Foi adotado um intervalo de 2 minutos entre as séries (Tabela 1) com o objetivo de evitar um processo precoce de exaustão. Este protocolo foi adaptado do estudo de Hortobágyi et al. (1996). O protocolo envolveu um aumento progressivo do volume de séries ao longo do mês e redução do volume no início de cada novo mês, diminuindo assim a sobrecarga a fim de possibilitar a adaptação muscular.

\section{Protocolo de avaliação}

Os sujeitos foram avaliados somente no membro inferior direito, pré e pós-treinamento excêntrico. $\mathrm{O}$ $\mathrm{GC}$ foi submetido às mesmas avaliações que o GE. $\mathrm{O}$ torque foi mensurado por meio de três contrações voluntárias máximas (CVM's) concêntricas nas velocidades angulares de $60 \%$ s e $420 \%$ s e excêntricas de $-60 \% \mathrm{~s} \mathrm{e}-300 \% \mathrm{~s}$ dos músculos extensores e flexores do joelho. O pico de torque foi selecionado na contração de maior valor para cada grupo muscular. O protocolo de avaliação foi randomizado e observou um intervalo de 2 minutos entre as contrações máximas a fim de evitar os efeitos da fadiga.

Um dinamômetro isocinético (Cybex, modelo NORM; Lumes \& Co., Ronkonkoma, Nova Iorque, EUA) foi utilizado para a avaliação do torque. Previamente ao início das avaliações os sujeitos foram submetidos a um período de aquecimento e alongamento da musculatura flexora e extensora do joelho. $\mathrm{O}$ aquecimento foi realizado em um cicloergômetro por 5 minutos, a uma potência de

Tabela 1. Protocolo de treinamento excêntrico.

Table 1. Eccentric training protocol.

\begin{tabular}{cccccc}
\hline Mês & Semana & Sessões & Séries & Repetições & $\begin{array}{c}\text { Volume } \\
\text { total }\end{array}$ \\
\hline \multirow{4}{*}{$\mathbf{1}^{\mathbf{o}}$} & $1^{\mathrm{a}}$ & 2 & 2 & 7 & 14 \\
& $2^{\mathrm{a}}$ & 3 & 3 & 7 & 21 \\
& $3^{\mathrm{a}}$ & 3 & 3 & 8 & 24 \\
& $4^{\mathrm{a}}$ & 3 & 3 & 9 & 27 \\
\hline \multirow{3}{*}{$\mathbf{2}^{\mathbf{o}}$} & $5^{\mathrm{a}}$ & 3 & 3 & 7 & 21 \\
& $6^{\mathrm{a}}$ & 3 & 4 & 7 & 28 \\
& $7^{\mathrm{a}}$ & 3 & 4 & 8 & 32 \\
& $8^{\mathrm{a}}$ & 3 & 4 & 9 & 36 \\
\hline \multirow{3}{*}{$\mathbf{3}^{\mathbf{o}}$} & $9^{\mathrm{a}}$ & 3 & 4 & 7 & 28 \\
& $10^{\mathrm{a}}$ & 3 & 5 & 7 & 35 \\
& $11^{\mathrm{a}}$ & 3 & 5 & 8 & 40 \\
& $12^{\mathrm{a}}$ & 3 & 5 & 9 & 45 \\
\hline
\end{tabular}

60 watts. Todos os sujeitos realizaram alongamento ativo dos músculos flexores e extensores do joelho durante 20 segundos antes e após a realização das avaliações.

Os sujeitos foram posicionados sentados, com a extremidade distal do membro inferior direito fixada ao braço mecânico do equipamento. $\mathrm{O}$ eixo de rotação do equipamento foi alinhado com o eixo de rotação aparente da articulação do joelho direito. Estímulo verbal foi fornecido a todos os sujeitos durante o protocolo a fim de garantir que os mesmos realizassem esforço máximo ao longo de todo o protocolo.

\section{Análise de dados}

$\mathrm{O}$ valor de torque mais elevado dentre as três contrações máximas realizadas por cada sujeito em cada velocidade angular foi escolhido para análise estatística.

\section{Análise estatística}

Todos os dados foram descritos por média e desvio padrão das razões de torque I:Q. O teste de ShapiroWilk e o teste de Mauchly foram utilizados para verificar a normalidade e a esfericidade dos dados, respectivamente. Para os dados que não apresentaram esfericidade foi utilizado o fator de correção de Greenhouse-Geisser. O teste ANOVA two-way (grupo, avaliações) para medidas repetidas, com post-hoc de Bonferroni, foi utilizado para as comparações entre os grupos e entre as avaliações. O teste $t$ de Student para dados dependentes foi utilizado na comparação entre os valores de torque (extensores e flexores) pré e pós-treinamento excêntrico. O software SPSS 18.0 for Windows foi utilizado para todas as análises, com um nível de significância de $\alpha=0,05$.

\section{Resultados}

Houve um aumento nas razões de toque I:Q concêntricas $\mathrm{GE}\left(\mathrm{I}_{\text {con }}: \mathrm{Q}_{\text {con }}\right)$ foi observado na velocidade angular de $420 \%$ do período pré $(0,43 \pm 0,14)$ para o pós-treinamento excêntrico $(0,53 \pm 0,16)(\mathrm{p}=0,036)$. Contudo na velocidade angular de $60 \%$ s do período pré $(0,47 \pm 0,11)$ para o pós $(0,53 \pm 0,09)$, treinamento excêntrico, não houve diferença significativa entre as avaliações, $p>0,05$ (Figura 1). Por outro lado para as razões excêntricas $\left(I_{\text {exc }}: Q_{\text {exc }}\right)$ houve uma redução nas razões de torque de I:Q do GE na velocidade de $-60^{\circ}$ s na comparação pré $(0,57 \pm 0,09)$ com pós $(0,48 \pm 0,07)$ treinamento excêntrico $(p=0,022)$, entretanto em altas velocidades excêntricas $\left(-300^{\circ} / \mathrm{s}\right)$, não foi encontrado diferença significativa entre o pré $(0,55 \pm 0,14)$ e pós $(0,63 \pm 0,13)$ treinamento excêntrico, $p>0,05$ (Figura 2). 


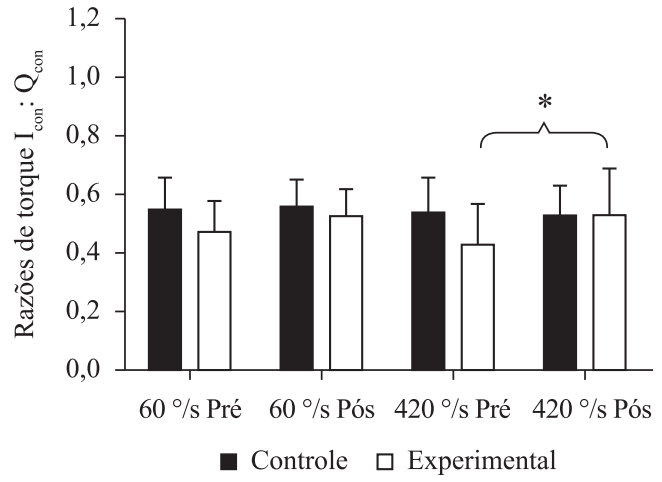

Figura 1. Razões de torque concêntrico. * = diferença significativa entre avaliações $(\mathrm{p}<0,05)$.

Figure 1. Concentric torque ratios. $*$ = significant difference between assessments $(p<0,05)$.

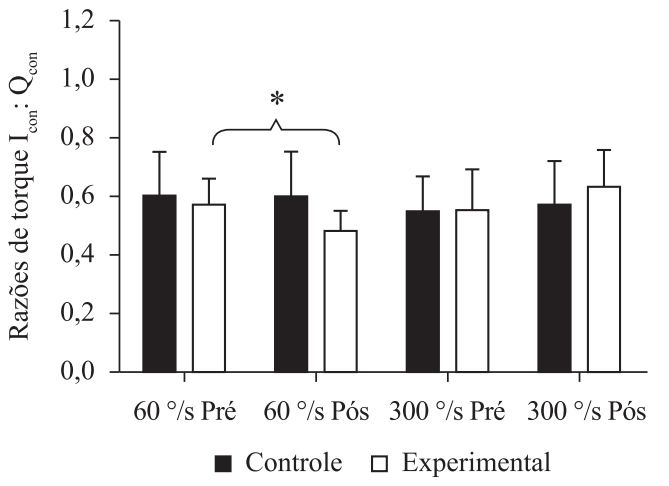

Figura 2. Razões de torque excêntrico. * = diferença significativa entre avaliações $(\mathrm{p}<0,05)$.

Figure 2. Eccentric torque ratios. * = significant difference between assessments $(p<0,05)$

Tabela 2. Diferença percentual do torque entre o pré e o pós-treinamento excêntrico.

Table 2. Percent difference in torque between the pre and post eccentric training.

\begin{tabular}{|c|c|c|c|c|}
\hline \multirow{3}{*}{ Velocidades } & \multicolumn{2}{|c|}{ Controle (dif. \% torque) } & \multicolumn{2}{|c|}{ Experimental (dif. \% torque) } \\
\hline & Extensores & Flexores & Extensores & Flexores \\
\hline & Média \pm DP & Média \pm DP & Média \pm DP & Média \pm DP \\
\hline$-300 \% / \mathrm{s}$ & $5,35 \pm 17,53$ & $8,39 \pm 22,41$ & $4,82 \pm 19,42$ & $25,69 \pm 36,78$ \\
\hline$-60 \% / \mathrm{s}$ & $5,53 \pm 8,19$ & $7,21 \pm 13,64$ & $48,47 \pm 19,44 *$ & $24,27 \pm 22,65^{*}$ \\
\hline $60 \% \mathrm{~s}$ & $-2,93 \pm 9,81$ & $1,06 \pm 13,57$ & $2,59 \pm 10,26$ & $25,16 \pm 53,19$ \\
\hline $420^{\circ} / \mathrm{s}$ & $10,79 \pm 22,42$ & $11,51 \pm 24,36$ & $26,85 \pm 40,49$ & $64,00 \pm 64,10^{*}$ \\
\hline
\end{tabular}

* = diferença significativa do percentual de torque entre as avaliações pré e pós $(\mathrm{p}<0,05)$.

$*$ = significant difference of torque percentual between pre and post assessments $(p<0,05)$.

O GC não apresentou nenhuma diferença significativa nas razões de torque velocidade I:Q avaliadas $(\mathrm{p}>0,05)$.

O GE apresentou a diferença percentual do torque bruto do pré para o pós-treinamento excêntrico, há incremento do percentual do torque dos músculos extensores $(48,47 \pm 19,44 \%)$ e flexores $(24,27 \pm 22,65 \%)$ do joelho na velocidade angular excêntrica de $-60 \%$, já na velocidade concêntrica de $420 \%$ s somente o grupo muscular flexor do joelho teve aumento percentual do torque $(64,00 \pm 64,10 \%)$ $\mathrm{p}<0,05$. Por outro lado o $\mathrm{GC}$ não apresentou alterações do percentual do torque bruto para os músculos extensores e flexores do joelho $\mathrm{p}>0,05$, (Tabela 2).

\section{Discussão e Conclusão}

O aumento das razões $I_{\text {con }}: Q_{\text {con }}$, está relacionado com um maior ganho de torque concêntrico dos músculos isquiotibiais em relação ao músculo quadríceps na velocidade de $420 \%$ s. Este ganho de torque percentual teve incremento de $27 \%$ para o músculos extensores do joelho e de $64 \%$ para os músculos flexores do joelho, alterando as razões de torque concêntricas em altas velocidades angulares. $\mathrm{O}$ aumento das razões
$\mathrm{I}_{\text {con }}: \mathrm{Q}_{\text {con }}$ em altas velocidades pode ser explicado pelo arranjo dos fascículos musculares. Como os músculos isquiotibiais apresentam uma área de secção transversa intermediária e menor ângulo de penação de suas fibras, além de fibras longas, esse grupo muscular apresenta grande eficiência em produzir força a maiores velocidades angular (Lieber, 1992; Lieber e Fridén, 2000).

Já nas razões $I_{\text {exc }}: Q_{\text {exc }}$, encontramos uma redução desses valores. Esta redução está relacionada com um maior ganho de torque excêntrico dos músculos extensores do joelho (48\%), em relação aos músculos flexores do joelho (24\%) na velocidade excêntrica de $-60 \%$ s (Tabela 2). A redução das razões $I_{\text {exc }}: Q_{\text {exc }}$ a $-60 \%$ s pode ter sido influenciada pela especificidade do treinamento excêntrico, que teria contribuído para as mudanças nas razões de torque na mesma velocidade do treino (Lieber, 1992). Entretanto, se isso fosse verdade o mesmo deveria ter ocorrido no caso das contrações concêntricas, o que não foi observado.

Talvez a explicação mais apropriada para os resultados tenha relação direta com a estrutura muscular. A sobrecarga mecânica máxima imposta aos grupos musculares antagonistas com o treinamento excêntrico provocou maior efeito no ganho de torque 
excêntrico nos músculos extensores do joelho. Isto se justifica pela morfologia do grupo muscular que apresenta fibras curtas com maiores ângulos de penação, além de maior área de secção transversa, em relação ao grupo muscular flexor do joelho. Devido a essas características morfológicas, o grupo muscular extensor do joelho apresenta grande eficiência na produção de força em menores velocidades angulares (Lieber, 1992; Lieber e Fridén, 2000) e a sobrecarga mecânica excêntrica parece ter produzido melhores resultados adaptativos na velocidade de treinamento.

Essas alterações nas razões de torque também foram encontradas em estudos prévios (Kannus, 1998; Lanferdini et al., 2010; Lephart et al., 2001), indicando que o treinamento excêntrico provocou desequilíbrio muscular entre os antagonistas do joelho na velocidade de treinamento, resultando no afastamento das razões de torque dos valores próximos de equilíbrio muscular entre os grupos musculares antagonistas do joelho (Aagaard et al., 1995; Impellizzeri et al., 2008).

Assim o treinamento excêntrico em uma baixa velocidade angular $(-60 \%$ s) aumentou as razões de torque $\mathrm{I}_{\text {con }}: \mathrm{Q}_{\text {con }}$ em altas velocidade $\left(420^{\circ} / \mathrm{s}\right)$, bem como reduziu as razões $I_{\text {exc }}: Q_{\text {exc }}$ a velocidades baixas $\left(60^{\circ} / \mathrm{s}\right)$, prejudicando o equilíbrio muscular entre grupos musculares antagonistas na velocidade de treinamento.

Muitos estudos tem investigado os efeitos do treinamento excêntrico na capacidade de produção de torque, seja em diferentes velocidades angulares (Farthing e Chilibeck, 2003; Paddon-Jones et al., 2001) ou mesmo, em uma velocidade fixa para diferentes grupos musculares Aagaard et al. (1996) encontraram redução da razão de torque $I_{\text {exc }}: Q_{\text {exc }}$ na velocidade de treinamento $\left(-30^{\circ} / \mathrm{s}\right)$. Estes resultados vão ao encontro dos resultados deste estudo onde também foi observado redução das razões na velocidade de treinamento $\left(-60^{\circ} / \mathrm{s}\right)$. O mecanismo mais aceito para estas adaptações neuromusculares, esta relacionados às diferentes arquiteturas musculares dos grupos musculares quadríceps e isquiotibiais que por sua vez, provocariam ganhos de força diferentes após um período de treinamento (Lieber e Fridén, 2000).

Futuras investigações sobre parâmetros intrínsecos musculares como o uso de técnicas de imagem como a ultra-sonografia (arquitetura muscular) podem possibilitar a identificação de adaptações estruturais na arquitetura muscular e podem possibilitar melhor explicar as possíveis alterações provocadas pelo treinamento excêntrico na estrutura muscular e, conseqüentemente, na capacidade de produção de força da musculatura envolvida pelo treinamento. Além disso, a investigação do efeito de diferentes protocolos de treinamento com diferentes sobrecargas mecânicas externas impostas a diferentes grupos musculares pode auxiliar na determinação de programas de treinamento e de reabilitação adequados às necessidades de grupos específicos submetidos a programas de treinamento excêntrico.

\section{Agradecimentos}

Os autores gostariam de agradecer aos colegas do Grupo de Pesquisa em Biomecânica e Cinesiologia (GPBiC) pela participação como sujeitos do estudo, e as agências de fomento em pesquisa Coordenação de Aperfeiçoamento de Pessoal de Nível Superior (CAPES) e Conselho Nacional de Pesquisa (CNPq) pelo auxílio financeiro para o desenvolvimento deste estudo.

\section{Referências}

AAGAARD, P.; SIMONSEN, E. B.; MAGNUSSON, S. P.; LARSSON, B.; DYHRE-POULSEN, P. A new concept for isokinetic hamstring:quadriceps muscle strength ratio. American Journal of Sports Medicine, v. 26, n. 2, p. 231-237, 1998. PMid:7484168. http://dx.doi. org/10.1111/j.1748-1716.1995.tb09927.x

AAGAARD, P.; SIMONSEN, E. B.; TROLLE, M.; BANGSBO, J.; KLAUSEN, K. Isokinetic hamstring/quadriceps strength ratio: influence from joint angular velocity, gravity correction and contraction mode. Acta Physiologica Scandinavia, v. 154, n. 4, p. 421-427, 1995.

AAGAARD, P.; SIMONSEN, E. B.; TROLLE, M.; BANGSBO, J.; KLAUSEN, K. Specificity of training velocity and training load on gains in isokinetic knee joint strength. Acta Physiologica Scandivica, v. 156, n. 2, p. 123-129, 1996. PMid:8868268. http://dx.doi.org/10.1046/j.1365201X.1996.438162000.x

ALBERT, M. Treinamento excêntrico em esportes e reabilitação São Paulo: Ed. Manole, 2002.

APPEN, L.; DUCAN, P. W. Strength Relationship of the Knee Musculature: Effects of Gravity and Sport. The Journal of Orthopaedic and Sports Physical Therapy, v. 7, n. 5, p. 231-235, 1986.

FARTHING, J. P.; CHILIBECK, P. D. The effects of eccentric and concentric training at different velocities on muscle hypertrophy. European Journal of Applied Physiology, v. 89 , n. 6 , p. 578-586, 2003. PMid:12756571. http://dx.doi. org/10.1007/s00421-003-0842-2

HEIDERSCHEIT, B. C.; SHERRY, M. A.; SILDER, A.; CHUMANOV, E. S.; THELEN, D. G. Hamstring Strain Injuries: Recommendations for Diagnosis, Rehabilitation and Injury Prevention. Journal of Orthopaedic and Sports Physical Therapy, v. 40, n. 2, p. 67-81, 2010.

HORTOBÁGYI,T.; HOUMARD, J. A.; FRASER, D. D.; DUDEK, R.; LAMBERT, N. J.; ISRAEL, R. G. Adaptive responses to muscle lengthening and shortening in humans. Journal of Applied Physiology, n. 80, p. 765-72, 1996.

IMPELLIZZERI, F. M.; BIZZINI, M.; RAMPININI, E.; CEREDA, F.; MAFFIULETT, N. A. Reliability of isokinetic 
strength imbalance ratios measured using the Cybex Norm dynamometer. Clinical Physiology and Functional Imaging, v. 28, n. 2, p. 113-119, 2008. PMid:18070123. http://dx.doi.org/10.1111/j.1475-097X.2007.00786.x

JONSSON, P.; ALFREDSON, H. Superior results with eccentric compared to concentric quadriceps training in patients with jumper's knee: a prospective randomized study. British Journal of Sports Medicine, v. 39, n. 11, p. 847-850, 2005. PMid:16244196. PMCid:1725058. http://dx.doi. org/10.1136/bjsm.2005.018630

KANNUS, P. Ratio of hamstring to quadriceps femoris muscles' strength in the anterior cruciate ligament insufficient knee: relationship to long-term recovery. Physical Therapy, v. 68 , n. 6 , p. $961-965,1988$.

LANFERDINI, F. J.; ROCHA, C. S. S.; FRASSON, V. B.; VAZ, M. A. Influência do treinamento excêntrico nas razões de torque de flexores/extensores do joelho. Fisioterapia e Pesquisa, v. 17, n. 1, p. 40-45, 2010.

LEPHART, S. M.; FERRIS, C. M.; RIEMANN, B. L.; FU, F. H. Gender differences in strength and lower extremity kinematics during landing. Clinical Orthopaedics and Related Research, v. 401, p. 162-169, 2001. PMid:12151893. http://dx.doi.org/10.1097/00003086200208000-00019

LIEBER, R. L. Skeletal muscle structure and function: implications for rehabilitation and sports medicine. Ed. Baltimore, Willians \& Wilkins, 1992.
LIEBER, R. L.; FRIDÉN, J. Functional and clinical significance of skeletal muscle architecture. Muscle Nerve, v. 23, n. 11, p. 1647-66, 2000. http://dx.doi.org/10.1002/10974598(200011)23:11\%3C1647::AID-MUS1\%3E3.0.CO;2-M

MICHAUT, A.; POUSSON, M. Eccentric contraction-induced mechanical and neurophysiological adaptations: the protective effect. Science \& Sports, v. 9, n. 6, p. 286-95, 2004. http://dx.doi.org/10.1016/j.scispo.2004.06.001

O'SULLIVAN, K.; O'CEALLAIGH, B.; O'CONNELL, K.; SHAFAT, A. The relationship between previous hamstring injury and the concentric isokinetic knee muscle strength of Irish Gaelic footballers. BioMed Central Musculoskeletal Disorders, v. 9, n. 30, p. 1471-2474, 2008.

PADDON-JONES, D.; LEVERITT, M.; LONERGAN, A.; ABERNETHY, P. Adaptation to chronic eccentric exercise in humans: the influence of contraction velocity. European Journal of Applied Physiology, v. 85, n. 5, p. 466-471, 2001. PMid:11606016. http://dx.doi.org/10.1007/s004210100467

PULL, M. R.; RANSON, C. Eccentric muscle actions: implications for injury prevention and rehabilitation. Physical Therapy in Sport, v. 8, n. 2, p. 88-97, 2007. http://dx.doi.org/10.1016/j.ptsp.2006.11.005

SEGER, J. Y.; THORSTENSSON, A. Effects of eccentric versus concentric training on thigh muscle strength and EMG. International Journal of Sports Medicine, v. 26, n. 1, p. 45-52, 2005. PMid:15643534. http://dx.doi. org/10.1055/s-2004-817892

\footnotetext{
Autores

Julio Cézar Lima da Silva, Lucas Coelho Job, Fábio Juner Lanferdini, Clarice Sperotto dos Santos Rocha, Marco Aurélio Vaz

Grupo de Pesquisa em Biomecânica e Cinesiologia - GPBiC, Laboratório de Pesquisa do Exercício - LAPEX, Universidade Federal do Rio Grande do Sul - UFRGS, Rua Felizardo, 750, CEP 90690-200, Porto Alegre, RS, Brasil
} 